\section{Fiberoptic LARYNGOSCOPE}

\section{DEAR SIR}

I am writing to comment on the paper "An introduction to the fiberoptic laryngoscope" recently published in the Journal (28: 475-478, 1981). While it is a well written article with excellent illustrations, I found it somewhat misleading and incomplete. The value of the fiberoptic bronchoscope in the management of difficult and potentially dangerous airway problems is firmly established, ${ }^{1,2}$ as stated in the abstract by Dr. Witton. However, his method, as attested by the cookbook recipe summary, is only applicable to elective cases, where no airway problems are apparent. In these situations intubation under direct laryngoscopy is not only convenient but speedy. As suggested, the technique described would indeed serve as a guideline for beginners, but Dr. Witton failed to advise the readers that this would by inappropriate or even dangerous for difficult airway problems where fiberoptic endoscopic intubation is truely indicated. In a series of fiberoptic bronchoscopic intubations that I have performed recently, his technique would have been universally contraindicated. For examples, two cases were patients with unstable cervical fractures held by halo-traction apparatus where extension of the head was impossible and dangerous; another case was a patient with a frozen temporomandibular joint where administration of thiopentone and succinylcholine may not be safe. All these patients were successfully intubated under minimal sedation and local anaesthesia. It is common knowledge that muscle relaxants are generally contraindicated when there are potential upper airway problems and yet these are often the cases where fiberoptic endoscope intubations may be life-saving.

Furthermore, as cautioned by Dr. Witton, there is a genuine risk of hypoxia developing during manipulation of the fiberoptic bronchoscope. Finally, the fiberoptic laryngoscope described in the article was an early model without suction capability, and as such fits Dr. Witton's quote "It looks great when you try it on the mannequins but it's sure no good for real people"; for real people have secretions, mucus, and often blood, any of which easily cloud the vision and make intubation impossible.

The fiberoptic bronchoscope is a valuable adjunct and its use in anaesthetic practice needs to encouraged. However, this must be done in a proper manner, the discussion of which is not possible in this letter, and no cookbook approach would suffice.

\author{
A.M. Lam, M.D., F.R.C.P.(C), \\ Department of Anaesthesia, \\ University Hospital and \\ University of Western Ontario.
}

\section{REFERENCES}

1. TAYLOR, P.A. \& TOWEY, R.M. The bronchofiberscope as an aid to endotracheal intubation. Brit. J. Anaesth. 44: 611-612 (1972).

2. Messeter, K.H. \& Petersson, K.I. Endotracheal intubation with the fiberoptic bronchoscope. Anaesthesia 35: 294-298 (1980).

\section{Hazard of Blocked Scavenge Valve}

\section{DeAR SIR,}

Flowerdew's report on "A hazard of scavenger port design"1 reminds us that with many of the earlier interim designs of scavenging equipment now in use we exchanged a potential long term hazard for acute life threatening hazards which provoked Miller and Cullen to write "The Cost of Scavenging - Is it worth it?"2 The following episode illustrates the hazards of a suction scavenging system.

The patient was intubated and connected to the breathing system of an Ohio 2000 anaesthetic apparatus fitted with Ohio diaphragm type scavenge valve \#209-8172-800 and a 1975 pattern Ohio suction gas evacuation system. At first nothing untoward happened but then it was noticed that the breathing bag continued to distend. Adjustment of the scavenge valve had no effect, the positive pressure within the system continued to increase. The patient was disconnected from the $Y$ piece and given mouth to tube respiration while the scavenging system was checked. The surplus gas conduit was disconnected from the scavenge valve and a finger inserted into the valve verified the free motion of the diaphragm valve. Yet, when the system was reassembled and the patient reconnected, the valve still would not release and pressure built up in the system once more. At this point the conduit was disconnected from the scavenge valve and at once the diaphragm valve functioned normally releasing the surplus gas from the system. It was clear that a blockage exited downstream of the valve.

Examination of the rest of the system at the rear of the gas machine disclosed that someone had taped over the open end of the corrugated 
tubing reservoir because they felt that gas was leaking out! As a result the vacuum system built up sufficient negative pressure to hold the diaphragm valve securely on its seating, preventing the escape of any gas. Under these circumstances other designs of scavenge valve permit the high negative pressure to empty the breathing system of gas after which a negative pressure of up to -380 torr can be exerted within the patient's thorax. ${ }^{3}$ The result would be equally lethal. These hazards were pointed out by Sharrock and Leith ${ }^{3}$ in 1977 and were well known to the manufacturer and anaesthesiologist members of the Z79 writing committee who produced the draft standard Z79.11 on Anesthesia Gas Pollution Control. ${ }^{4}$ This standard requires that an interface system with negative and positive pressure limiting valves be provided between the vacuum source and the scavenging valve to prevent such an occurrence.

Unfortunately, like ourselves, most hospitals have the earlier designed equipment which lacks this vital protection. As suitable interface units are now available from most manufacturers which will prevent the development of dangerously high or low pressures within the system, surely we should review the safety of our presently used scavenging apparatus and add negative and positive pressure limiting interface systems?

Leslie Rendell-Baker, M.D.,

Professor of Anesthesiology,

Loma Linda University,

Loma Linda, Califomia 92350, U.S.A.

\section{REFERENCES}

1. Flowerdew, R.M. A hazard of scavenger port design. Can. Anaesth. Soc. J. 28: 481-483 (1981).

2. Miller, M.G. \& Cullen, B.F. The cost of scavenging - Is it worth it? Anesth. Analg. 58 265-266 (1979).

3. Sharrock, N.E. \& Leith, D.E. Potential pulmonary barotrauma when venting anesthetic gases to suction. Anesthesiology 46: 152-154 (1977).

4. Z79.11.1982 American National Standard for Anesthesia Gas Pollution Control. ANSI, 1430 Broadway, New York, NY 10018.

\section{UNSUSPECTED PhaEOChROMOCYTOMA}

DEAR SIR,

I am writing to comment on the paper "Unsuspected Phaeochromocytoma Presenting During Surgery" by Doctors L. Wooster and R.I.
Mitchell, which appeared in the September 1981 issue of this journal $(28: 471-474,1981)$.

Several discrepancies are obvious in this paper. The authors stated that "during the procedure the patient's blood pressure rose from $140 / 80$ to $200 / 100$." Figure 1, however, indicates that the blood pressure rose initially to $225 / 230$ systolic. This appears to have occurred 7.5 minutes from the beginning of the anaesthetic. The authors further stated that "Halothane ... was administered to lower the blood pressure towards its preoperative level." Figure 1 indicates that the blood pressure never fell lower than $190 \mathrm{~mm} \mathrm{Hg}$ systolic (much higher than the preoperative pressure of $130 \mathrm{~mm} \mathrm{Hg}$ systolic). Furthermore, "at the end of the procedure ... he was stable at his preoperative haemodynamic values." Again, Figure 1 illustrates that the blood pressure at this time was actually $200 \mathrm{~mm}$ $\mathrm{Hg}$ systolic. One also questions what the diastolic pressures were during the course of this procedure.

The authors also mention that concomitant with the sudden rise in blood pressure, the pulse rose to 130 beats per minute. However, it did not fall below 100 beats per minute during the anaesthetic course. And it was stated that the 45-minute procedure "was done under general anaesthesia using ... Innovar $2 \mathrm{ml} . "$ However, only $1 \mathrm{ml}$ appears to have been given according to the Figure.

This paper adequately relates the proper management of a patient who has sustained a myocardial infarction. As the authors stated in their discussion, myocardial infarctions have previously been reported to occur in patients with a phaeochromocytoma undergoing general anaesthesia.

It has been well documented that if a sudden, unexplained, sustained rise in pulse and blood pressure occurs during an anesthetic administration, one should always suspect a phaeochromocytoma. The procedure should then be terminated as quickly as possible, and a cause for this increase by investigated. ${ }^{1,2}$ As indicated in this paper, this massive rise occurred 7.5 minutes into the anaesthetic. One questions, then, why the procedure was allowed to progress for 37.5 minutes under such unusual circumstances.

The authors do not mention whether this massive elevation of blood pressure and pulse occurred in relationship to any external stimulus such as intubation, surgical incision, too light anaesthesia, or other factors. If the only temporal relationship to the elevation in blood pressure 